\title{
c-ANCA-induced neutrophil-mediated lung injury: a model of acute Wegener's granulomatosis
}

\author{
K. Hattar*, S. Oppermann*, C. Ankele*, N. Weissmann\#, R.T. Schermuly\#, \\ R.M. Bohle ${ }^{\star}$, R. Moritz ${ }^{+}$, B. Krögel*, W. Seeger ${ }^{\#}$, F. Grimminger*, \\ U. Sibelius* and U. Grandel*
}

ABSTRACT: Anti-neutrophil cytoplasmic antibodies (C-ANCA) targeting proteinase 3 (PR3) are implicated in the pathogenesis of Wegener's granulomatosis (WG). Fulminant disease can present as acute lung injury (ALI).

In this study, a model of ALI in WG was developed using isolated rat lungs. Isolated human polymorphonuclear leukocytes (PMNs) were primed with tumour necrosis factor (TNF) to induce surface expression of PR3.

Co-perfusion of TNF-primed neutrophils and monoclonal anti-PR3 antibodies induced a massive weight gain in isolated lungs. This effect was not observed when control immunoglobulin G was co-perfused with TNF-primed PMNs. The c-ANCA-induced oedema formation was paralleled by an increase in the capillary filtration coefficient as a marker of increased pulmonary endothelial permeability. In contrast, pulmonary artery pressure was not affected. In the presence of the oxygen radical scavenger superoxide dismutase and a NADPH oxidase inhibitor, c-ANCAinduced lung oedema could be prevented. Inhibition of neutrophil elastase was equally effective in preventing c-ANCA-induced lung injury.

In conclusion, anti-PR3 antibodies induced neutrophil mediated, elastase- and oxygen radicaldependent $A L I$ in the isolated lung. This experimental model supports the hypothesis of a pathogenic role for C-ANCA in WG and offers the possibility of the development of therapeutic strategies for the treatment of lung injury in fulminant WG.

KEYWORDS: Acute lung injury, anti-neutrophil cytoplasmic antibodies, neutrophil activation, Wegener's granulomatosis

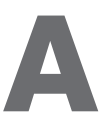

cute lung injury (ALI) is a rare but lifethreatening complication of Wegener's Granulomatosis (WG) [1]. In WG, ALI usually occurs together with acute glomerulonephritis as pulmonary-renal syndrome, and is characterised by diffuse alveolar haemorrhage with oedema formation and extravasation of blood components, mainly erythrocytes and neutrophils, into the alveolar space [2]. Histologically, ALI in WG is defined by pulmonary capillaritis with disruption of pulmonary endothelial integrity as a putative underlying pathophysiological mechanism [3].

Activation and transmigration of neutrophils are thought to play a major role in the development of ALI [4] and in some experimental models the severity of ALI is diminished upon neutrophil depletion [5]. Neutrophil-derived mediators implicated in the pathogenesis of ALI are reactive oxygen species (ROS), proteases and cationic proteins, as well as pro-inflammatory cytokines, such as tumour necrosis factor (TNF) $[6,7]$. These mediators were also found to be elevated in the circulation and, in case of myeloperoxidase (MPO) and peroxidase, in the bronchoalveolar fluid (BALF) of patients with active WG. The levels of these neutrophil-derived products were shown to correlate with clinical disease activity [8-10]. Moreover, circulating neutrophils display an activated phenotype in active WG [11] and neutrophil depletion substantially diminished the severity of experimentally induced anti-neutrophil cytoplasmic autoantibody (ANCA)-associated vasculitis [12]. Thus, activated neutrophils seem to be major effector cells in the development of inflammatory tissue injury associated with WG.

The mechanisms of neutrophil activation in WG have been extensively studied over the past
AFFILIATIONS

*Medical Clinic V,

\#Medical Clinic II, University of Giessen Lung Center (UGLC)

Giessen, and

"Dept of Pathology, University of Saarland, Homburg-Saar, Germany. +Mayo Clinic, Dept of Physiology and Biomedical Engineering, Rochester, MN, USA.

CORRESPONDENCE

K. Hattar

University of Giessen Lung Center (UGLC)

Medical Clinic V

Justus-Liebig University Giessen

Klinikstrasse 36

D-35392

Germany

E-mail: katja.hattar@innere.med.unigiessen.de

Received:

Sept 182008

Accepted after revision:

Dec 092009

First published online:

Dec 232009 
decades, and, apart from their outstanding role as diagnostic markers, circulating "classic" anti-neutrophil cytoplasmic autoantibodies (c-ANCAs) targeting the neutrophil granule protein proteinase-3 (PR3) have been attributed with pathogenic potential in the development of inflammatory vascular lesions in WG, as they can activate neutrophils in vitro [13]. In neutrophils, the auto-antigen PR3 is expressed on the cell surface in response to priming with pro-inflammatory cytokines such as TNF [14]. Binding of anti-PR3 antibodies to neutrophils activates a wide variety of inflammatory cellular functions, such as respiratory burst, degranulation and the release of lipid mediators and cytokines [15-17]. In leukocyte-endothelial co-cultures, ANCA-activated polymorphonuclear leukocytes (PMNs) adhere to and lyse endothelial cells $[18,19]$. While ANCAs targeting MPO have recently been shown to cause vasculitis and glomerulonephritis in an animal model [20], little is known about the pathogenic role of anti-PR3 antibodies in the development of pulmonary lesions in active WG. Against this background, the aim of the present study was to clarify whether PR3 ANCAs are involved in the pathogenesis of pulmonary dysfunction in WG. In essence, it was found that PR3 ANCAs potently activate neutrophils in the pulmonary circulation of isolated rat lungs resulting in the development of pulmonary oedema. Oedema formation was attributable to neutrophil release of elastase and oxygen radicals in response to anti-PR3 antibodies. Thus, a pathogenic role of PR3 ANCA in lung injury in WG is suggested.

\section{MATERIALS AND METHODS}

\section{Materials}

Sterile Krebs-Henseleit-hydroxyethylamylopectin buffer (KHB) was obtained from Serag-Wiessner (Naila, Germany). The buffer contained $120 \mathrm{mM} \mathrm{NaCl}, 4.3 \mathrm{mM} \mathrm{KCl}, 1.1 \mathrm{mM} \mathrm{KH}_{2} \mathrm{PO}_{4}$, $24 \mathrm{mM} \mathrm{NaHCO}, 2.4 \mathrm{mM} \mathrm{CaCl}, 1.3 \mathrm{mM} \mathrm{MgCl} 2$ and $2.4 \mathrm{~g} \cdot \mathrm{L}^{-1}$ of glucose as well as $5 \%$ (weight/volume) hydroxyethylamylopectin (molecular weight 2000.000) as an oncotic agent. $\mathrm{O}_{2}$, $\mathrm{CO}_{2}$, and $\mathrm{N}_{2}$ were obtained from Messer Griesheim (Herborn, Germany).

Ficoll-Paque was purchased from Pharmacia (Upsalla, Sweden). Superoxide dismutase (SOD), AAPVKC and isotype control mouse immunoglobulin (Ig)G $\mathrm{G}_{1}$ (MOPC-21) were obtained from Sigma (Deisenhofen, Germany), while recombinant human TNF- $\alpha$ was from R\&D Systems (Wiesbaden, Germany). The NADPH oxidase inhibitor diphenylene iodonium (DPI) was obtained from Merck (Schwalbach, Germany), while the 5-lipoxygenase (5-LO)-activation inhibitor MK-886 was from Biomol (Hamburg, Germany).

PBS was obtained from Gibco Laboratories (Grand Island, NY, USA). All other biochemicals were obtained from Merck (Darmstadt, Germany).

The murine monoclonal anti-PR3 antibody 4A5 (mouse IgG) was purchased from Wieslab AG (Lund, Sweden), while the rabbit polyclonal anti-human MPO antibody (DAKO A398) used for histological staining was from DAKO (Hamburg, Germany). Phycoerythrin (PE)-conjugated goat-anti-mouse IgG was from Dako, and pooled human IgG (Octagam) was from Octapharma (Langenfeld, Germany).

\section{Isolation of human neutrophils}

Neutrophils were isolated from venous blood of healthy donors by centrifugation over a Ficoll-Paque gradient as previously described [17]. In brief, EDTA-anticoagulated blood was layered over Ficoll-Paque and centrifuged at $400 \times g$ for $35 \mathrm{~min}$. After removal of mononuclear cells, erythrocytes were sedimented in $1 \%$ polyvinyl alcohol. Residual erythrocytes were removed by hypotonic lysis, cells were washed twice in $\mathrm{Ca}^{2+} / \mathrm{Mg}^{2+}$-free PBS, and resuspended in PBS containing $\mathrm{CaCl}_{2}$ $(1 \mathrm{mM})$ and $\mathrm{MgSO}_{4}(1 \mathrm{mM})$ at $10^{7} \mathrm{PMN} \cdot \mathrm{mL}^{-1}$. Cell purity was $>97 \%$, as quantified by flow cytometry, and cell viability was $>96 \%$, as assessed by trypan blue dye exclusion. Prior to addition to the lung perfusate, neutrophils were treated with TNF (2 ng $\left.\cdot \mathrm{mL}^{-1}\right)$ for $30 \mathrm{~min}$ to induce surface expression of PR3.

\section{Flow cytometry}

For the determination of surface expression of PR3, flow cytometry was performed. In brief, isolated neutrophils $\left(5 \times 10^{6} \mathrm{~mL}^{-1}\right)$ were incubated with TNF $\left(2 \mathrm{ng} \cdot \mathrm{mL}^{-1}\right)$ or shamincubated for $30 \mathrm{~min}$. Cells were pelleted at $4^{\circ} \mathrm{C}$, and resuspended in PBS containing $0.1 \%$ bovine serum albumin and $0.02 \%$ sodium azide. Then, $2 \times 10^{5}$ cells were distributed to each well of flexible round bottom microtitre plates and washed. Prior to the addition of monoclonal antibodies, $20 \mu \mathrm{L}$ of pooled human immunoglobulin $\left(100 \mu \mathrm{g} \cdot \mathrm{mL}^{-1}\right)$ was added to block Fc $\gamma$ receptors. Murine monoclonal anti-PR3 antibodies $\left(10 \mu \mathrm{g} \cdot \mathrm{mL}^{-1}\right)$ or mouse control $\operatorname{IgG}\left(10 \mu \mathrm{g} \cdot \mathrm{mL}^{-1}\right)$ were then added, and incubated for $30 \mathrm{~min}$ at $4^{\circ} \mathrm{C}$. After three washes, the secondary antibody, a PE-conjugated goat-anti-mouse IgG $\left(50 \mu \mathrm{g} \cdot \mathrm{mL}^{-1}\right)$ was added and again incubated for $30 \mathrm{~min}$ at $4^{\circ} \mathrm{C}$. After three final washes, cells were resuspended in PBS and kept on ice until flow cytometric analysis. Flow cytometry was performed in a FACScan (Becton-Dickinson, Mountain View, CA, USA) using forward and orthogonal light scatter to select viable cells. Software CellQuest ${ }_{\circledR}$ research software (BectonDickinson) was used to analyse the generated data.

\section{Lung isolation and perfusion}

The model of the isolated rat lung has already been described in detail [21, 22]. In brief, male CD rats (body weight 350$450 \mathrm{~g}$ ) were deeply anaesthetised with sodium pentobarbital (100 $\mathrm{mg} \cdot \mathrm{kg}^{-1}$ body weight i.p.). After local anaesthesia with $2 \%$ xylocaine and median incision, the trachea was dissected and a tracheal cannula was immediately inserted. A median laparotomy was performed and subsequently the rats were anticoagulated with 1,000 units of heparin. Mechanical ventilation was started with $5.3 \% \mathrm{CO}_{2}, 21 \% \mathrm{O}_{2}$ and $73.7 \% \mathrm{~N}_{2}$ (tidal volume $4 \mathrm{~mL}$, frequency $65 \mathrm{~min}^{-1}$, end expiratory pressure $2 \mathrm{cmH}_{2} \mathrm{O}$ ) using a small animal respirator KTR-4 (Hugo Sachs Electronic, March-Hugstetten, Germany). After midsternal thoracotomy, the right ventricle was incised, a cannula was fixed in the pulmonary artery, and the apex of the heart was cut off to allow pulmonary venous outflow. Simultaneously pulsatile perfusion with KHB containing 5\% hydroxyethyl starch was performed and the lungs were carefully excised and placed in a supine position. A cannula was fixed through the left ventricle in the left atrium to obtain a closed perfusion circuit without leakage. After extensive rinsing of the vascular bed, the lungs were perfused with a pulsatile flow of $13 \mathrm{~mL} \cdot \mathrm{min}^{-1}$ (total volume $150 \mathrm{~mL}$ ) in a recirculating model. The left atrial pressure was set at $2 \mathrm{mmHg}$ under baseline conditions 
(0 referenced at the hilum) and the whole perfusion system was equilibrated at $37.5^{\circ} \mathrm{C}$. Lungs selected for the study were those that: 1) had a homogenous white appearance without signs of haemostasis or oedema formation; 2) had pulmonary artery and ventilation pressures in the normal range; and 3) were isogravimetric during a steady state period of $30 \mathrm{~min}$.

The pulmonary artery pressure $(P \mathrm{pa})$, pulmonary venous pressure $(P \mathrm{pv})$ and the weight of the isolated organ were registered continuously, with $P$ pa being a direct measure of pulmonary vascular resistance.

The venous pressure was repeatedly elevated by sequential hydrostatic challenge of $10 \mathrm{cmH}_{2} \mathrm{O}$ for $8 \mathrm{~min}$. After the steady state period, lung weight was set at zero and lung weight gain was calculated as the weight difference before and after each hydrostatic challenge manoeuvre.

The capillary filtration coefficient was calculated from the slope of weight gain, as described previously [23].

\section{Experimental protocols}

Lungs without any drug application were perfused under standard conditions for $180 \mathrm{~min}$. After a control hydrostatic challenge (30 min after the initiation of perfusion) TNF-primed neutrophils were added to the perfusate at a final concentration of $10^{6} \mathrm{~mL}^{-1}$. When indicated, anti-PR3 antibodies (4A5, $10 \mu \mathrm{g} \cdot \mathrm{mL}^{-1}$ ) or equal amounts of isotype-matched control IgG (MOPC-21) were co-perfused with human neutrophils. All pharmacological agents (DPI, SOD, MK-886 and AAPVKC) were admixed to the perfusate before the first hydrostatic challenge, and were re-administered prior to the third hydrostatic challenge.

\section{Histological examination}

At the end of the perfusion period, one of each rat lungs were fixed by instillation with formaldehyde solution (4.5\%, $\mathrm{pH} 7.0$ ) via the main bronchi. Fixation was allowed to proceed overnight at room temperature. Subsequently, tissues were dissected and routinely embedded in paraffin and sections of $5 \mu \mathrm{m}$ were stained with haematoxylin-eosin. The other lungs were snap-frozen in liquid nitrogen after instillation with OCT (Tissue Tek, Sakura, Japan) via the main bronchi. Frozen tissues were stored at $-80^{\circ} \mathrm{C}$. A few minutes before the preparation of cryosections using a cryomicrotome (Leica Jung CM 3000, Bensheim, Germany) the lung tissues were dissected into smaller tissue blocks and $5 \mu \mathrm{m}$ cryosections were prepared. After drying at room temperature overnight, these sections were stored at $-20^{\circ} \mathrm{C}$ for a few days and stained with anti-MPO $(1: 6,000)$ using the APAAP ChemMate kit (K5000, DAKO). Haematoxylin was used for counterstaining. A Zeiss Axioskop 40 (Carl Zeiss, Jena, Germany) with PlanNEO Fluar $20 \times / 0.5$ NA objective lenses, in connection with a JVC KY-S75U digital camera (JVC, Friedberg, Germany) was used to acquire the micrographs. Images were processed with Diskus acquisition software, version 4.5 (Hilgers, Königswinter, Germany). A neutrophil aggregate was defined as intravascular neutrophil accumulation reaching an extent of more than tree diameters of alveolar septa in at least one dimension. Quantitation was done in duplicate by two blinded reviewers (R.M. Bohle and R. Moritz).

\section{Statistics}

For statistical comparison, ANOVA was performed, followed by Tukey's honestly significant difference test when appropriate. A level of $p<0.05$ was considered to be significant.

\section{RESULTS}

\section{Anti-PR3 antibodies induce neutrophil-dependent oedema} formation in isolated rat lungs

In order to determine neutrophil surface expression of PR3, a prerequisite for specific ANCA-targeting, neutrophils were subjected to flow cytometry after the isolation procedure. Freshly isolated neutrophils exhibited minor surface expression of the ANCA target antigen, while neutrophil priming with TNF ( $\left.2 \mathrm{ng} \cdot \mathrm{mL}^{-1}\right)$ induced marked surface expression of PR3, as assessed by flow cytometry (fig. 1).

Under baseline conditions, isolated rat lungs were perfused for $180 \mathrm{~min}$ and baseline lung weight remained stable over the entire perfusion period (control). Perfusion of TNF-primed human neutrophils $\left(10^{6} \mathrm{~mL}^{-1}\right)$ did not induce any weight gain, whereas after simultaneous perfusion of TNF-primed human neutrophils $\left(10^{6} \mathrm{~mL}^{-1}\right)$ and monoclonal anti-PR3 antibodies $\left(10 \mu \mathrm{g} \cdot \mathrm{mL}^{-1}\right)$ a massive pulmonary oedema formation, quantified as weight increase, developed (fig. 2). Significant weight gain was noted after 140 min of perfusion. Occasionally, the experiment was prematurely terminated due to massive fluid accumulation after $180 \mathrm{~min}$. In contrast, co-perfusion of isotype-matched control-IgG and TNF-primed human neutrophils did not exert any effect on pulmonary physiology. Likewise, perfusion of anti-PR3 antibodies in the absence of human neutrophils or in the presence of unprimed neutrophils (data not shown) was ineffective.

Histological and immunohistochemical examination of isolated lungs revealed the retention of human neutrophils in the isolated perfused rat lung. Neutrophil transmigration into the alveolar lumen was sometimes observed in lungs perfused with human neutrophils (fig. 3). However, there was no differences in the degree of neutrophil infiltration between anti-PR3 and control IgG perfusion (table 1).

\section{Oedema formation in anti-PR3-treated lungs is accompanied by microvascular leakage response}

The capillary filtration coefficient $\left(K_{\mathrm{f}, \mathrm{c}}\right)$, calculated from the slope of weight gain after stepwise elevation of the venous pressure, remained stable during the entire perfusion period in untreated lungs as well as upon perfusion with TNF-primed neutrophils $\left(10^{6} \mathrm{~mL}^{-1}\right)$. However, when anti-PR3 antibodies were co-perfused with human neutrophils, a dramatic increase in $K_{\mathrm{f}, \mathrm{c}}$ values paralleled oedema formation (fig. 4). Significant elevations of $K_{\mathrm{f}, \mathrm{c}}$ values were noted after perfusion of TNFprimed neutrophils and anti-PR3 antibodies. As anticipated from the lung weight data, perfusion of anti-PR3 antibodies alone, as well as co-perfusion of TNF-primed neutrophils and mouse control IgG, did not induce any alterations to the microvascular barrier function in isolated lungs.

\section{Ppa is not affected by anti-PR3 antibodies}

In control experiments, $P$ pa values were constant $(6-7 \mathrm{mmHg})$ throughout the entire experimental period. While the $P$ pa did rise slightly immediately after addition of human neutrophils, the $P$ pa remained stable over the 3 -h perfusion period and, 

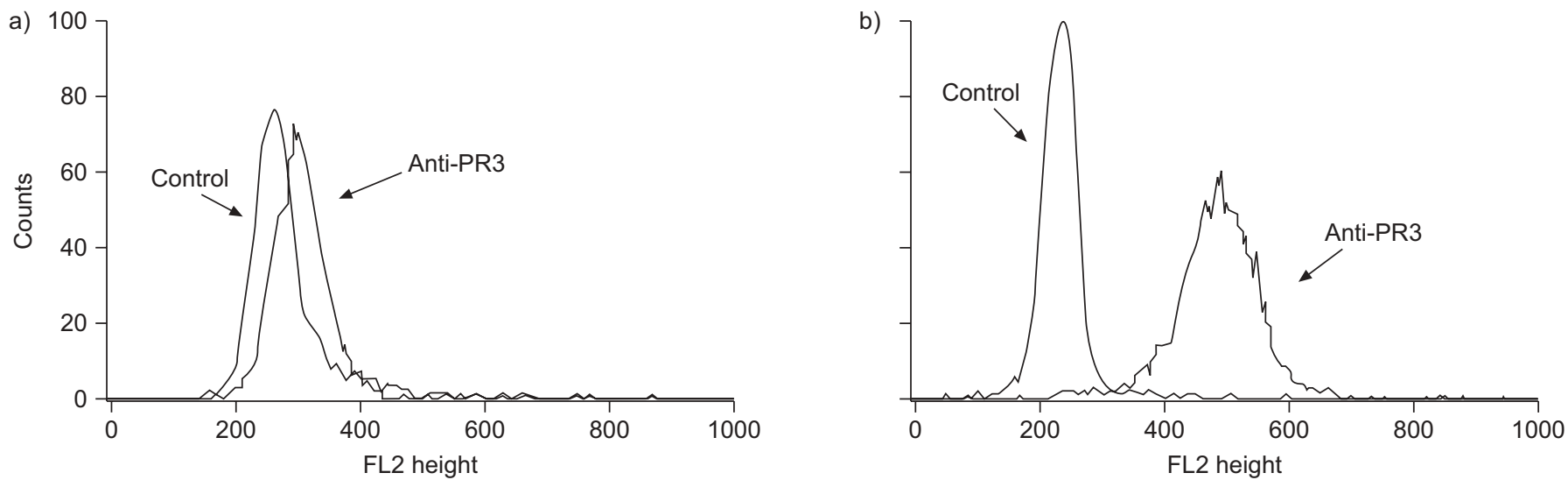

FIGURE 1. Surface expression of proteinase-3 (PR3) in isolated neutrophils in response to tumour necrosis factor (TNF). Isolated neutrophils $\left(5 \times 10^{6} \mathrm{~mL}^{-1}\right)$ were either a) sham-incubated or b) primed with TNF $\left(2 \mathrm{ng} \cdot \mathrm{mL}^{-1}\right)$ for $30 \mathrm{~min}$. Following incubation with isotype control immunoglobulin (Ig)G $\left(\mathrm{control}, 10 \mu \mathrm{g} \cdot \mathrm{mL}^{-1}\right)$ or anti-PR3 antibodies $\left(10 \mu \mathrm{g} \cdot \mathrm{mL}^{-1}\right)$, a phycoerythrin-labelled goat anti-mouse $\operatorname{lgG}\left(50 \mu \mathrm{g} \cdot \mathrm{mL}^{-1}\right)$ was added, and flow cytometry was performed. Note the strong staining for PR3 in TNFprimed versus unprimed polymorphonuclear leukocytes, as depicted in one out of four representative flow cytometric studies.

notably, co-perfusion of anti-PR3 antibodies or control IgG did not induce any significant changes in $P$ pa (fig. 5).

\section{Effect of oxygen radical scavengers on anti-PR3-induced pulmonary oedema formation}

In the presence of SOD $\left(300 \mathrm{U} \cdot \mathrm{mL}^{-1}\right)$ catalysing the dismutation from superoxide $\left(\mathrm{O}_{2}^{-}\right)$to hydrogen peroxide $\left(\mathrm{H}_{2} \mathrm{O}_{2}\right)$, the auto-antibody-induced weight increase was strongly attenuated (fig. 6a). The NADPH oxidase inhibitor DPI $\left(5 \mu \mathrm{g} \cdot \mathrm{mL}^{-1}\right)$ was equally effective as SOD, thus suggesting that neutrophil

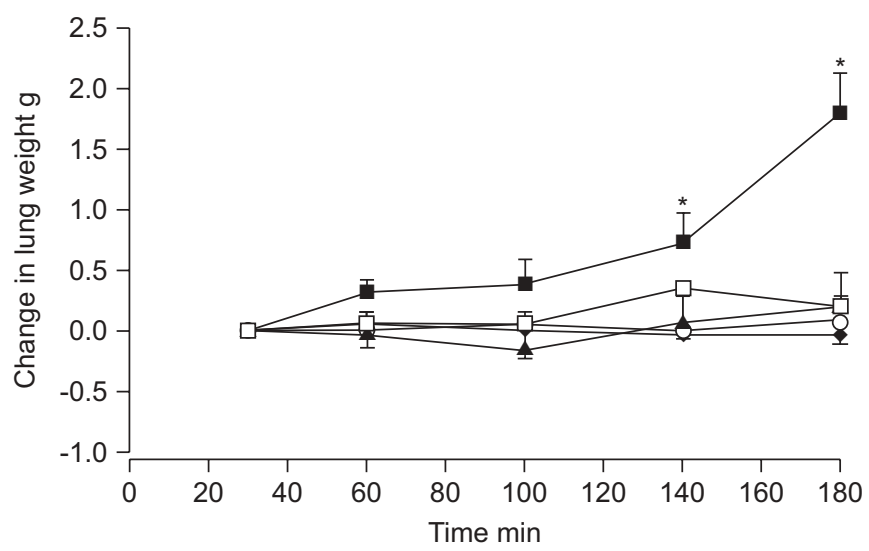

FIGURE 2. Effect of anti-proteinase-3 (anti-PR3) antibodies on lung weight in isolated rat lungs. After an isogravimetric steady-state period of $30 \mathrm{~min}$, a control hydrostatic challenge was performed, and lung weight was set to zero. Isolated rat lungs were either perfused with tumour necrosis factor (TNF)-primed neutrophils $\left(10^{6} \mathrm{~mL}^{-1}\right)$ alone $(\mathbf{\Lambda})$ or polymorphonuclear leukocytes (PMNs) were given together with murine monoclonal anti-PR3 antibodies $\left(10 \mu \mathrm{g} \cdot \mathrm{mL}^{-1}\right)(\boldsymbol{\square})$ or equal concentrations of an isotype-matched control immunoglobulin (lg) G ( $\square$ ). As a control group, lungs were buffer-perfused without the administration of human neutrophils ( ) over the entire perfusion period or anti-PR3-antibodies were perfused in the absence of neutrophils $(\bigcirc)$. Lung venous pressure elevations were performed at indicated time points. Changes in lung weight were quantified after each hydrostatic manoeuvre and are expressed as change from baseline. Data reflect mean \pm SEM of at least five independent experiments. ${ }^{*}: \mathrm{p}<0.05$ compared with controls, PMN group, PMN+lgG group and mono anti-PR3-perfused lungs.
NADPH oxidase was the source of superoxide in our model. Similarly impressive was the effect of these oxygen radical suppressors on the disturbed microvascular barrier function: in the presence of SOD, the elevation of $K_{\mathrm{f}, \mathrm{c}}$ values upon treatment of isolated lungs with anti-PR3 antibodies and TNFprimed neutrophils was nearly normalised, and the NADPH oxidase inhibitor was equally protective (fig. $6 \mathrm{~b}$ ).

\section{Role of neutrophil elastase, but not 5-LO products in anti- PR3-induced lung oedema formation}

As anti-PR3-induced pulmonary oedema formation was clearly dependent on the presence of neutrophils, the contribution of other neutrophil mediators to ALI was investigated. Inhibition of 5-lipoxygenase activation with MK-886 $(7.5 \mu \mathrm{M})$ did not prevent pulmonary oedema formation, as measured by changes in the weight of isolated lungs (fig. 7a) or increase in $K_{\mathrm{f}, \mathrm{c}}$ values (fig. $7 \mathrm{~b}$ ). Interestingly, both responses were strongly attenuated, and in case of $K_{\mathrm{f}, \mathrm{c}}$ normalised to control values, when the specific neutrophil elastase inhibitor AAPVKC $(5 \mu \mathrm{M})$ was co-perfused with neutrophils and anti-PR3 antibodies (fig. 7a and b). Combined inhibition of ROS by SOD and neutrophil elastase by AAPVKC had no additive or synergistic effect (data not shown).

\section{DISCUSSION}

Severe clinical presentations of WG, such as pulmonary-renal syndrome or isolated alveolar haemorrhage are characterised by the occurrence of ALI and require immediate treatment. Since anti-PR3 antibodies have been suggested to posses pathogenic potential in WG, the question was posed whether these auto-antibodies could induce lung injury.

Compelling evidence is provided in the present study documenting that ANCAs, targeting the neutrophil serine protease PR3, are pathogenic in an isolated rat lung model causing ALI. Upon perfusion of monoclonal anti-PR3 antibodies and TNF-primed human neutrophils in isolated rat lungs, an increase in lung vascular permeability occurred, which proceeded to severe vascular leakage and oedema formation. This effect was not attributable to enhanced capillary filtration pressure, as the $P$ pa remained stable despite 

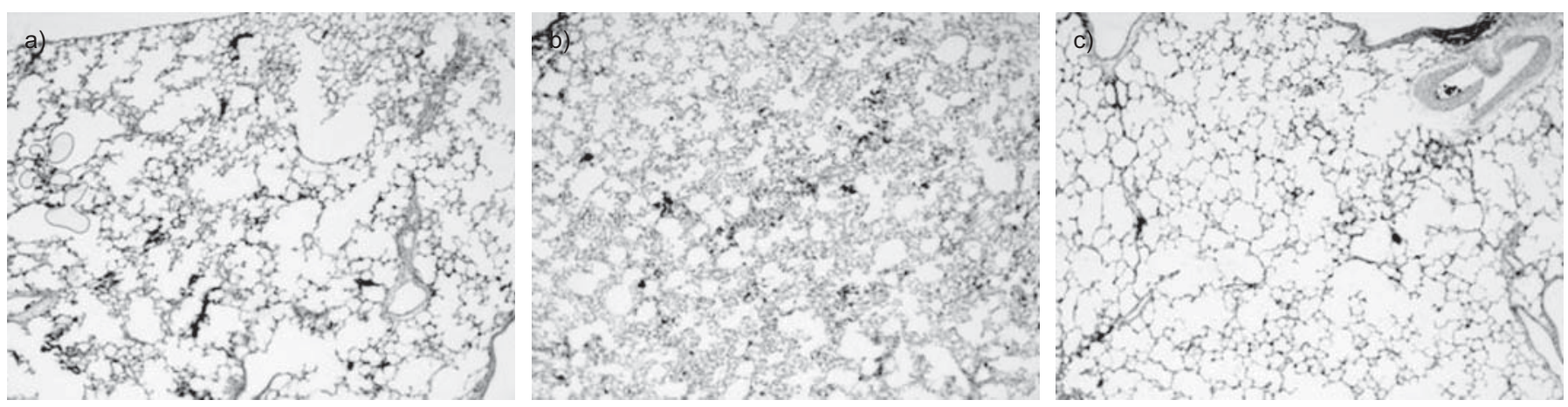

FIGURE 3. Accumulation of human neutrophils in isolated rat lungs. Isolated rat lungs were buffer-perfused (a) or perfusion with tumour necrosis factor (TNF)-treated human neutrophils $\left(10^{6} \mathrm{~mL}^{-1}\right)\left(\mathrm{b}\right.$ and c) was performed. Anti-proteinase-3 antibodies $\left(10 \mu \mathrm{g} \cdot \mathrm{mL}^{-1}\right)$ (b) or equal amounts of isotype-matched control immunoglobulin $\mathrm{G}$ (c) were co-perfused with human neutrophils. Repeatedly, hydrostatic challenges were performed by elevation of the venous pressure. At the end of the perfusion period of $180 \mathrm{~min}$, rat lungs were harvested. Paraffin-embedded tissues and OCT-embedded frozen tissues of superior lobe were stained with haematoxylin-eosin; additionally, paraffin-embedded tissue sections were stained with anti-myeloperoxidase/haematoxylin. Note the accumulation of human neutrophils in the alveolar capillaries of rat lungs $(b$ and $c)$, as depicted for representative $(n=3)$ immunostained slides of each group. Original magnification $\times 2.5$

anti-PR3 challenge. Anti-PR3-induced lung injury was strictly dependent on the presence of target-antigen expressing, preactivated human neutrophils, and was caused by neutrophil oxygen radical formation and elastase degranulation, whereas 5-LO-metabolites were not involved.

The isolated lung model was selected to investigate the pathogenic role of anti-PR3 antibodies for several reasons. First, although an elegant model for anti-MPO-associated renal vasculitis has been established [20], no valid animal model for pulmonary WG exists. Secondly, the lungs are, apart from the

\begin{tabular}{lcc} 
TABLE 1 & $\begin{array}{l}\text { Semiquantitative analysis of human neutrophil } \\
\text { retention in isolated rat lungs }\end{array}$ \\
\cline { 2 - 3 } & \multicolumn{2}{c}{ PMN aggregates per $\mathbf{~ m m}^{-\mathbf{2}}$} \\
\hline Reviewer $\mathbf{1}$ & Reviewer $\mathbf{2}$ \\
\hline Control & $0 \pm 0$ & $0 \pm 0$ \\
PMN and anti-PR3 & $10.33 \pm 3.95^{\star}$ & $15.00 \pm 6.6^{\star}$ \\
PMN and IgGc & $9.33 \pm 1.66^{*}$ & $12.33 \pm 2.68^{*}$ \\
\hline
\end{tabular}

Isolated rat lungs were buffer-perfused (control) or perfusion with tumour necrosis factor-treated human neutrophils $\left(10^{6} \mathrm{~mL}^{-1}\right)$ and anti-proteinase-3 (anti-PR3) antibodies or istotype-matched control immunoglobulin $(\mathrm{lg}) \mathrm{G}$ was performed. Repeatedly, hydrostatic challenges were performed by elevation of the venous pressure. At the end of the 180-min perfusion period, rat lungs were harvested. Paraffin-embedded tissues and OCT-embedded frozen tissues of superior lobe origin were stained with haematoxylin-eosin; additionally, paraffin-embedded tissues were stained with anti-myeloperoxidase/haematoxylin. Polymorphonuclear leukocyte (PMN) retention was expressed as number of neutrophil aggregates, which were defined as intravascular neutrophil accumulation reaching an extent of more than three diameters of alveolar septa in at least one dimension. Quantitation was done in duplicate from the upper lobe in an tissue area of $21.36 \mathrm{~mm}^{2}$ (rectangular area selected at low power view) by two blinded reviewers (R.M. Bohle and R. Moritz) The number of neutrophil aggregates is given per $\mathrm{mm}^{2}$ and values are expressed as mean \pm SEM $(n=3) .{ }^{*}: p<0.05$ compared with controls. No differences were obtained between the reviewers, or between the anti-PR3 and IgGc group. kidneys, the organ system most frequently affected in fulminant presentations of WG and, thirdly, the isolated lung model allows the registration of acute pathophysiological changes typical for ALI, such as oedema formation, and increases in vascular permeability or $P$ pa.

The experimental model facilitated the perfusion of isolated rat lungs with TNF-primed human neutrophils and the well defined and widely used mouse anti-human monoclonal antiPR3 antibody 4A5. The co-perfusion of human neutrophils as target cells for the murine monoclonal anti-PR3-antibody 4A5 was necessary, as mouse anti-human PR3 antibodies did not cross-react with rat neutrophils in previous studies [24, 25] and

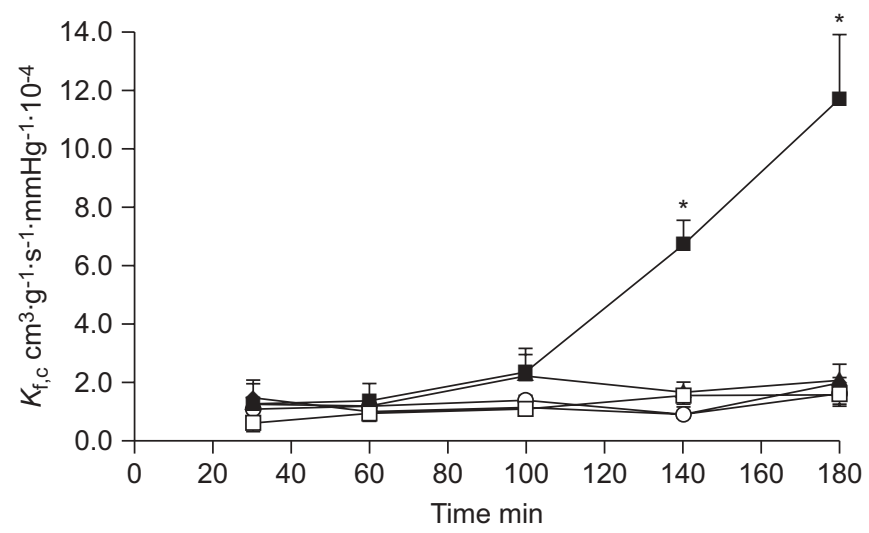

FIGURE 4. Effect of anti-proteinase-3 (anti-PR3) antibodies on microvascular leakage in isolated rat lungs. Following an isogravimetric steady state period of $30 \mathrm{~min}$, isolated rat lungs were either perfused with tumour necrosis factor-primed neutrophils $\left(10^{6} \mathrm{~mL}^{-1}\right)$ alone $(\mathbf{\Lambda})$ or polymorphonuclear leukocytes (PMNs) were given together with murine monoclonal anti-PR3 antibodies $\left(10 \mu \mathrm{g} \cdot \mathrm{mL}^{-1}\right)(\mathbf{\square})$ or equal concentrations of an isotype-matched control immunoglobulin $(\mathrm{lg}) \mathrm{G}(\square)$ alternatively, anti-PR3 antibodies were perfused in the absence of neutrophils $(\bigcirc)$. Controls represent buffer-perfused lungs $(\bullet)$. Lung venous pressure elevations for determination of the capillary filtration coefficient $\left(K_{\mathrm{f}, \mathrm{c}}\right)$ were performed at indicated time points. Data reflect mean \pm SEM of at least five independent experiments. *: $\mathrm{p}<0.05$ compared with controls, PMN group, PMN+lgG group and mono antiPR3-perfused lungs. 


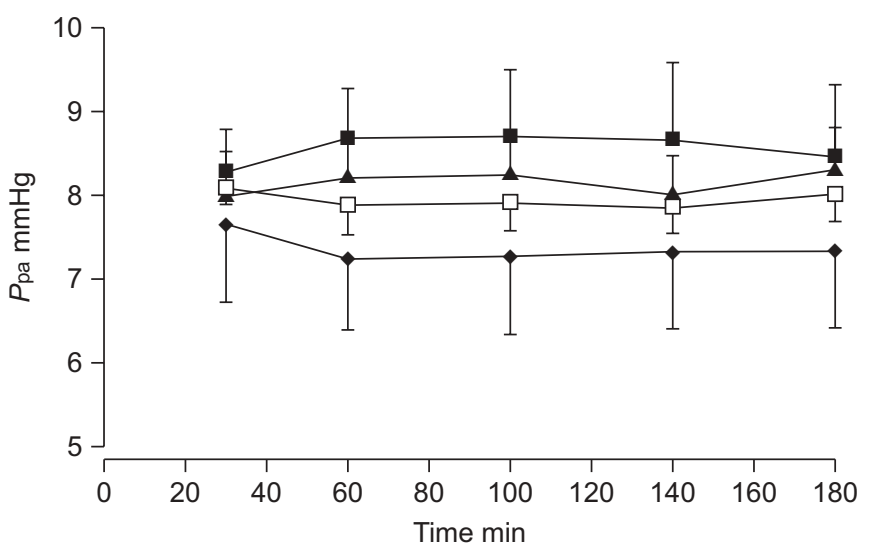

FIGURE 5. Effect of anti-proteinase-3 (anti-PR3) antibodies on the pulmonary artery pressure $(P \mathrm{pa})$ in isolated rat lungs. Isolated rat lungs were either bufferperfused for $180 \mathrm{~min}(\bullet)$ after a steady state period, or perfusion with tumour necrosis factor-primed human neutrophils $\left(10^{6} \mathrm{~mL}^{-1}\right)$ alone $(\mathbf{\Lambda})$ or in combination with murine monoclonal anti-PR3 antibodies $\left(10 \mu \mathrm{g} \cdot \mathrm{mL}^{-1}\right)$ (四) was performed. Alternatively, isotype-matched control immunoglobulin $\mathrm{G}\left(10 \mu \mathrm{g} \cdot \mathrm{mL}^{-1}\right)$ was perfused with polymorphonuclear leukocytes $(\square)$. The $P$ pa as a direct marker of vascular resistance was registered continuously. Data reflect mean \pm SEM of at least five independent experiments. No significant changes in Ppa were noted between the different experimental groups.

antibodies to rat PR3 are, as the state of our knowledge, not available. Clearly, the isolated lung model only reflects acute pathogenic effects of anti-PR3 antibodies and does not replace an animal model of anti-PR3-associated vasculitis. Recently, successful immunisation of rodents with chimeric human/ mouse PR3 and subsequent induction of an autoimmune response to mouse and rat PR3 has been performed, thus providing new perspectives to study the pathogenesis of antiPR3-associated vasculitis [25].

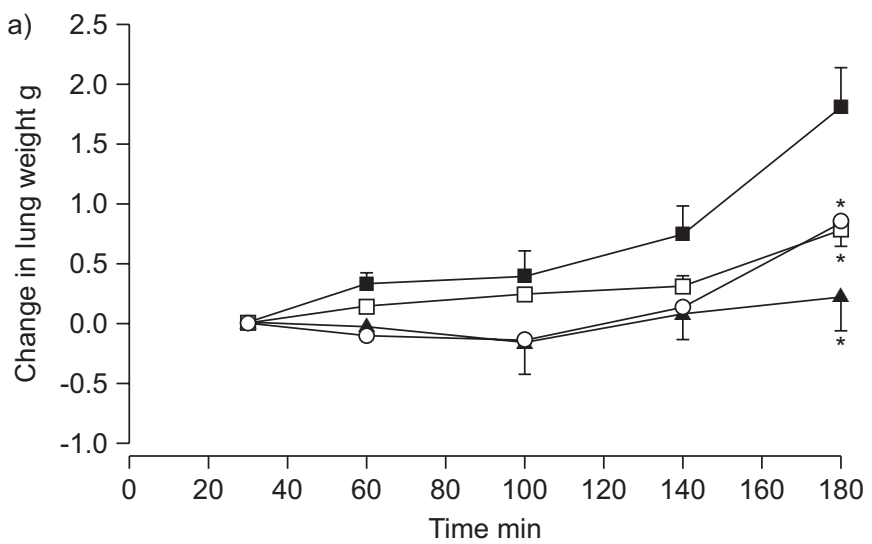

Human neutrophils have been used in various neutrophildependent models of organ injury in the rat [22, 26], and were currently shown to be effectively "trapped" in the alveolar capillaries of these animals. However, although only anti-PR3 antibodies, and not control-IgG-induced neutrophils mediated lung injury in the current model, we could not observe any morphological differences between anti-PR3 and control-IgG perfused lungs. This discrepancy might be due to the fact that the documentation of PMN infiltration, which did not differ in terms of quantity for both anti-PR3 and IgG perfused lungs, does not necessarily reflect any changes in the activation status of neutrophils, as also described for other models of lung injury [27]. The quantitation of in situ neutrophil activation, for example, by in situ detection of ROS or elastase degranulation, would be helpful in this context, but is beyond the scope of the current study. Moreover, severe damage to the structural integrity of the lungs cannot be expected to occur in the current short-time experiments and due to methodological limitations (for example, the use of a protein-free perfusate), oedema formation could only be assessed by physiological parameters, such as weight gain and increase in the capillary filtration coefficient, which was indeed specific for anti-PR3 antibodies.

The capacity of the anti-PR3 antibodies to induce lung injury was strictly dependent upon the presence of PR3-expressing, TNF-primed human neutrophils, as the antibody per se did not induce any changes in the isolated rat lung physiology over the entire perfusion period. Thus, any unspecific disruption of pulmonary microvascular integrity by the currently used antiPR3 antibody may be excluded. Moreover, neither perfusion of isotype-matched control-IgG and TNF-primed neutrophils did increase lung vascular permeability, nor did co-perfusion of anti-PR3 and unprimed neutrophils, which do not express surface PR3, as documented by the currently performed flow cytometric studies. Therefore, specific targeting of PR3, which was shown to be expressed on the surface of isolated leukocytes after TNF priming, in line with previous studies [14],

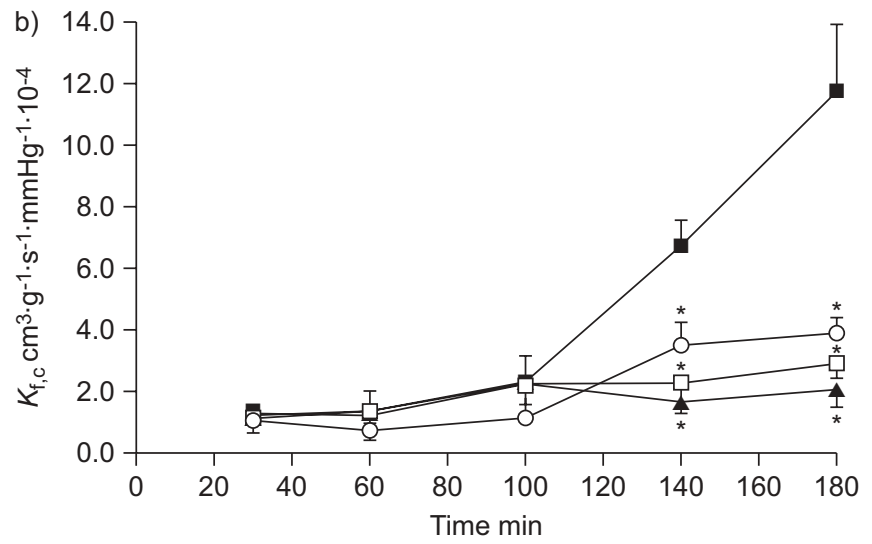

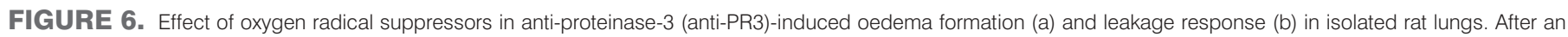

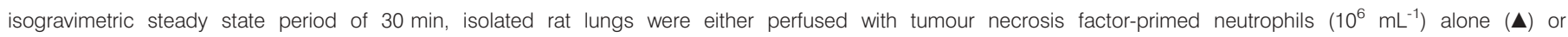

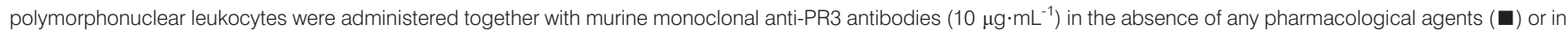

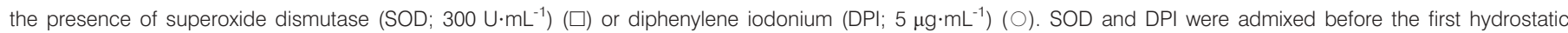

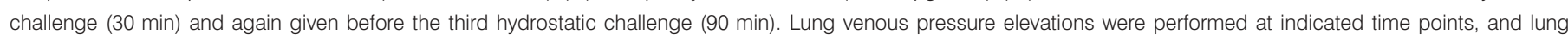

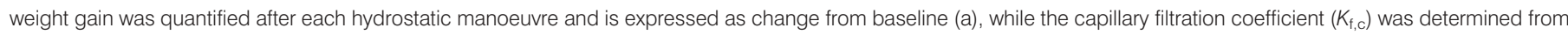

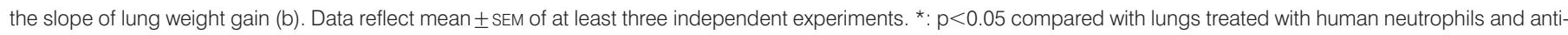
PR3 antibodies in the absence of any pharmacological agent. 

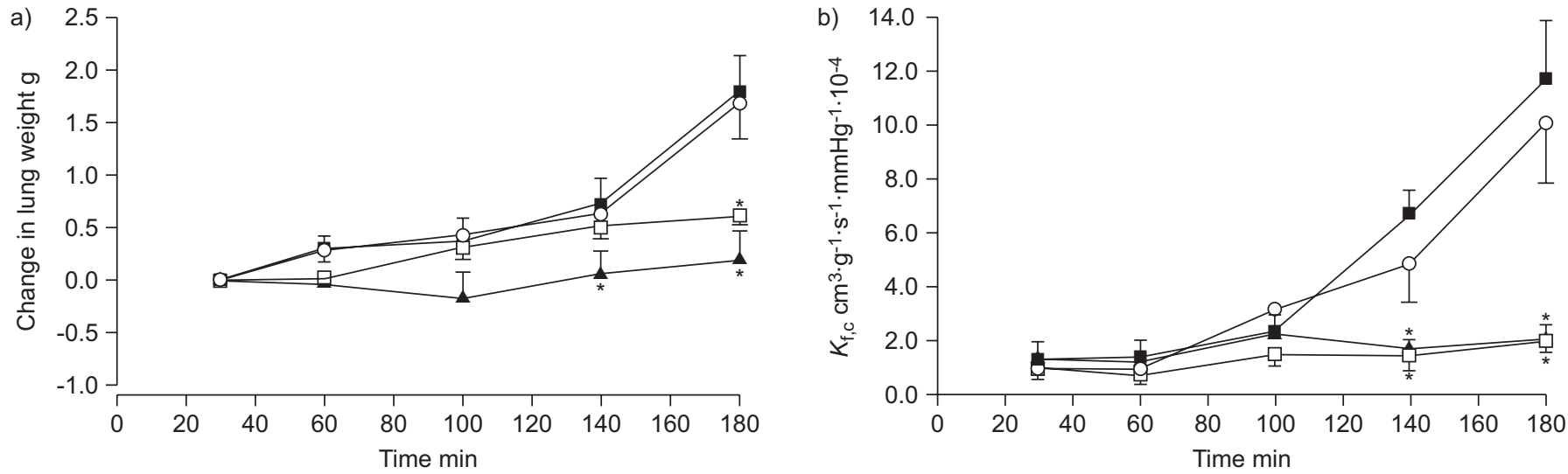

FIGURE 7. Effect of elastase and leukotriene inhibition in anti-proteinase-3 (anti-PR3)-induced oedema formation (a) and leakage response (b) in isolated rat lungs. Isolated rat lungs were either perfused with tumour necrosis factor-primed neutrophils $\left(10^{6} \mathrm{~mL}^{-1}\right)$ alone $(\mathbf{\Lambda})$ or polymorphonuclear leukocytes (PMNs) and murine monoclonal anti-PR3 antibodies $\left(10 \mu \mathrm{g} \cdot \mathrm{mL}^{-1}\right)$ in the absence of any pharmacological agents $(\mathbf{\square})$, or the elastase inhibitor AAPVKC $(5 \mu \mathrm{M})(\square)$ or the 5-lipoxygenase (5-LO) antagonist MK-886 $(7.5 \mu \mu \mathrm{M})(\bigcirc)$ were co-perfused with PMN and anti-PR3 antibodies. Both inhibitors were administered before the first, and again before the third, hydrostatic challenge. Hydrostatic challenges were repeatedly performed at indicated time points and lung weight gain was quantified after each hydrostatic manoeuvre and is expressed as change from baseline (a). b) The capillary filtration coefficient $\left(K_{\mathrm{f}, \mathrm{C}}\right)$ is given and expressed in $\mathrm{cm}^{3} \cdot \mathrm{g}^{-1} \cdot \mathrm{s}^{-1} \cdot \mathrm{mmHg}^{-1} \cdot 10^{-4}$. Data reflect mean $\pm \mathrm{SEM}$ of at least three independent experiments. *: $p<0.05$ compared with lungs treated with human neutrophils and anti-PR3 antibodies in the absence of elastase or 5-LO inhibition.

and the subsequent initiation of multiple, now well-characterised signalling events $[28,29]$, seem to underlie the ANCA-mediated lung injury. As the experiments were performed in the absence of complement sources, complement-dependent mechanisms, which have been implicated in many forms of antibodydependent leukocyte activation, including models of transfusionrelated ALI [30], were not operative in the anti-PR3-associated lung injury.

Although the exact molecular mechanisms of anti-PR3induced leukocyte activation are not completely understood, anti-PR3-related lung injury was clearly caused by ROS formation, as well as elastase liberation from anti-PR3activated neutrophils: in the presence of the oxygen radical scavenger SOD, catalysing the reduction from superoxide $\left(\mathrm{O}_{2}{ }^{-}\right)$ to hydrogen peroxide $\left(\mathrm{H}_{2} \mathrm{O}_{2}\right)$, the ANCA-induced oedema formation was completely reversed. Obviously, superoxide and not hydrogen peroxide is the primary oxygen metabolite responsible for the ANCA-mediated increase in vascular permeability. The fact that oedema formation could be prevented by the NADPH oxidase inhibitor DPI suggests that NADPH oxidases are the major source of superoxide release in ANCA-induced lung injury. Although DPI interferes with the mitochondrial superoxide production [31], the clear dependence of microvascular leakage on the presence of human neutrophils gives strong arguments for neutrophil NADPH oxidase as the source of superoxide in anti-PR3-induced lung injury. Well in line with this reasoning, the capacity of anti-PR3 antibodies to induce NADPH oxidase-dependent neutrophil superoxide formation has been repeatedly proven in vitro [15, 16] and in vivo, the degree of tissue injury in WG correlates with the presence of toxic oxygen metabolites released by activated neutrophils [32]. Moreover, increased levels of markers of oxidative stress, such as exhalative $\mathrm{H}_{2} \mathrm{O}_{2}$ and blood lipid peroxidation levels, were found in patients with ALI [7].

Apart from oxygen radicals, elastase was apparently centrally involved in mediating ANCA-induced lung injury, as demonstrated by the protective effect of the highly specific elastase inhibitor AAPVCK [33]. A key role for neutrophil elastase has been postulated in models of lung injury due to its capacity to degrade extracellular matrix proteins and protease inhibitors [34]. A role for elastase in the pathogenesis of pulmonary injury in WG is well established: the protease has been found to be elevated in the BALF and in the circulation of patients with active disease $[8,10]$, is liberated from neutrophils upon stimulation with anti-PR3 antibodies $[15,17]$ and was recently found to mediate endothelial cell damage by ANCA-activated neutrophils in vitro [34], thus well in line with the present results.

Interestingly, inhibition of elastase or superoxide formation completely reversed the anti-PR3-induced lung injury and combined application of both inhibitors could not amplify this effect. These findings might be related to the previously postulated synergy between oxidants and neutrophil elastase in lung injury: while elastase was shown to increase oxygen radical formation in macrophages [35] and pulmonary epithelial cells [36], oxygen radicals were found to inactivate endogenous elastase inhibitors and to increase elastase toxicity in the isolated rat lung [37]. Thus, oxidant- and elastasemediated tissue destruction are obviously not independent events in this model of ANCA-induced lung injury either, and the simultaneous release of both mediators is mandatory for the effects observed.

It has been previously demonstrated that neutrophils release leukotrienes in response to stimulation with anti-PR3 antibodies [17], and 5-LO products have been shown to be involved in leukocyte-mediated pulmonary injury [30]. However, a highly specific inhibitor of 5-LO activation did not exert any protective effects in anti-PR3-induced lung injury here. Thus, leukotrienes do not contribute to tissue injury in this model. This might be related to the fact that leukotriene generation is critically dependent on the presence of the substrate arachidonic acid, which was not provided in the 
present studies. Although a role for leukotrienes in mediating ANCA-induced tissue injury cannot be ruled out in vivo, these mediators do not contribute to ALI under the experimental conditions employed.

In conclusion, auto-antibodies targeting neutrophil PR3 may be crucially involved in the development of lung injury in WG. Synergistic effects of anti-PR3-induced neutrophil oxygen radical formation and elastase degranulation were identified as central mechanisms mediating microvascular damage. Pharmacological interference with these mediators might be protective in acute lung injury in WG.

\section{SUPPORT STATEMENT}

This work was supported by the Deutsche Forschungsgemeinschaft (SFB 547/B8, SFB 547/Z1) and by the Excellence Cluster Cardiopulmonary System (DFG).

\section{STATEMENT OF INTEREST}

Statements of interest for N. Weissmann, R.T. Schermuly and F. Grimminger can be found at www.erj.ersjournals.com $/ \mathrm{misc} /$ statement.dtl

\section{ACKNOWLEDGEMENTS}

We would like to thank R. Morty (Dept of Internal Medicine, UGLC, University of Giessen, Giessen, Germany) for carefully reviewing the manuscript.

\section{REFERENCES}

1 Brusselle G. Pulmonary renal syndromes. Acta Clin Belg 2007; 62: 88-96.

2 Specks U. Diffuse alveolar hemorrhage syndromes. Curr Opin Rheumatol 2001; 13: 12-17.

3 Gaudin PB, Askin FB, Falk RJ, et al. The pathologic spectrum of pulmonary lesions in patients with anti-neutrophil cytoplasmic autoantibodies specific for anti-proteinase 3 and anti-myeloperoxidase. Am J Clin Pathol 1995; 104: 7-16.

4 Reutershan J, Ley K. Bench to bedside review: acute respiratory distress syndrome - how neutrophils migrate into the lung. Crit Care 2004; 8: 453-461.

5 Chignard M, Balloy V. Neutrophil recruitment and increased permeability during acute lung injury induced by lipopolysaccharide. Am J Physiol 2000; 279: L1083-L1090.

6 Moraes TJ, Zurawska JH, Downey GP. Neutrophil granule contents in the pathogenesis of lung injury. Curr Opin Hematol 2006; 13: 21-27.

7 Ware LB. Pathophysiology of acute lung injury and the acute respiratory distress syndrome. Semin Respir Crit Care 2006; 27: 337-349.

8 Hoffman GS, Sechler JM, Gallin JI, et al. Bronchoalveolar lavage analysis in Wegener's granulomatosis. A method to study disease pathogenesis. Am Rev Respir Dis 1991; 143: 401-407.

9 Deguchi Y, Shibata N, Kishimoto S. Enhanced expression of the tumour necrosis factor/cachetin gene in peripheral blood mononuclear cells from patients with systemic vasculitis. Clin Exp Immunol 1990; 81: 311-314.

10 Haubitz M, Schulzeck P, Schellong S, et al. Complexed plasma elastase as an in vivo marker of leukocyte activation in antineutrophil cytoplasmic antibody associated vasculitis. Arthritis Rheum 1997; 1680-1684.

11 Schnabel A, Csernok E, Braun J, et al. Activation of neutrophils, eosinophils, and lymphocytes in the lower respiratory tract in Wegener's granulomatosis. Am J Respir Crit Care Med 2000; 161: 399-405.
12 Xiao $\mathrm{H}$, Heeringa $\mathrm{P}$, Liu $\mathrm{Z}$, et al. The role of neutrophils in the induction of glomerulonephritis by anti-myeloperoxidase antibodies. Am J Pathol 2005; 167: 39-45.

13 Kallenberg CG, Heeringa P, Stegeman CA. Mechansims of disease: pathogenesis and treatment of ANCA associated vasculitides. Nat Clin Pract Rheumatol 2006; 661-670.

14 Csernok E, Ernst M, Schmitt W, et al. Activated neutrophils express proteinase 3 on their plasma membrane in vitro and in vivo. Clin Exp Immunol 1994; 95: 244-250.

15 Falk RJ, Terrell RS, Charles LA, et al. Anti-neutrophil cytoplasmic autoantibodies induce neutrophils to degranulate and produce oxygen radicals in vitro. Proc Natl Acad Sci USA 1990; 87: 4115-4119.

16 Kettritz R, Jennette JC, Falk RJ. Crosslinking of ANCA antigens stimulates superoxide release by human neutrophils. J Am Soc Nephrol 1997; 8: 386-394.

17 Grimminger F, Hattar K, Papavassilis C, et al. Neutrophil activation by anti-proteinase 3 antibodies in Wegener's granulomatosis: role of exogenous arachidonic acid and leukotriene $\mathrm{B}_{4}$ generation. J Exp Med 1996; 184: 1567-1572.

18 Savage COS, Pottinger BE, Gaskin G, et al. Autoantibodies developing to myeloperoxidase and proteinase 3 in systemic vasculitis stimulate neutrophil cytotoxicity towards cultured endothelial cells. Am J Pathol 1992; 141: 335-342.

19 Radford DJ, Savage CO, Nash B. Treatment of rolling neutrophils with antineutrophil cytoplasmic antibodies causes conversion to firm integrin-mediated adhesion. Arthritis Rheum 2000; 43: 1337-1345.

20 Xiao $\mathrm{H}$, Heeringa $\mathrm{P}, \mathrm{Hu} \mathrm{P}$, et al. Anti-neutrophil cytoplasmic autoantibodies specific for myeloperoxidase cause glomerulonephritis and vasculitis in mice. J Clin Invest 2002; 110: 955-963.

21 Ermert L, Ermert M, Althoff A, et al. Vasoregulatory prostanoid generation proceeds via cyclooxygenase-2 in noninflamed rat lungs. J Pharmacol Exp Ther 1998; 286: 1309-1314.

22 Sachs U, Hattar K, Weissmann N, et al. Antibody-induced neutrophil activation as a trigger for transfusion-related acute lung injury in an ex vivo rat lung model. Blood 2006; 107: 1217-1219.

23 Seeger W, Walmrath D, Grimminger F, et al. Adult respiratory distress syndrome: model systems using isolated perfused rabbit lungs. Methods Enzymol 1994; 233: 549-584.

24 Garwicz D, Lindmark A, Hellmark T, et al. Characterization of the processing and granular targeting of human proteinase 3 after transfection of the rat RBL or the murine 32D leukemic cell lines. J Leukoc Biol 1997; 61: 113-123.

25 van der Geld YM, Hellmark T, Selga D, et al. Rats and mice immunized with chimeric human/mouse proteinase 3 produce autoantibodies to mouse PR3 and rat granulocytes. Ann Rheum Dis 2007; 66: 1679-1682.

26 Grandel U, Reutemann M, Kiss L, et al. Staphylococcal alpha-toxin provokes neutrophil-dependent cardiac dysfunction: role of ICAM-1 and cys-leukotrienes. Am J Physiol Heart Circ Physiol 2002; 282: H1157-H1165.

27 Minamiya $\mathrm{Y}$, Saito $\mathrm{H}$, takahashi $\mathrm{N}$, et al. Polymorphonuclear leukocytes are activated during atelectasis before lung reexpansion in rats. Shock 2008; 30: 81-86.

28 Hewins P, Williams JM, Wakelam MJ, et al. Activation of Syk in neutrophils by antineutrophil cytoplasm antibodies occurs via Fc $\gamma$ receptors and CD18. J Am Soc Nephrol 2004; 15: 796-808.

29 Kettritz R, Choi M, Butt W, et al. Phosphatidylinositol 3-kinase controls antineutrophil cytoplasmic antibodies-induced respiratory burst in human neutrophils. J Am Soc Nephrol 2002; 13: 17401749.

30 Seeger W, Schneider U, Kreusler B, et al. Reproduction of transfusion-related acute lung injury in an ex vivo lung model. Blood 1990; 76: 1438-1444. 
31 Li Y, Trush MA. Diphenyleneiodonium, an NAD(P)H oxidase inhibitor, also potently inhibits mitochondrial reactive oxygen species production. Biochem Biophys Res Commun 1998; 18: 295-299.

32 Brouwer E, Huitema MG, Mulder AHL, et al. Neutrophil activation in vitro and in vivo in Wegener's granulomatosis. Kindey Int 1994; 45: 1120-1131.

33 Zeiher BG, Matsuoka S, Kawabata K, et al. Neutrophil elastase and acute lung injury: prospects for sivelestat and other neutrophil elastase inhibitors as therapeutics. Crit Care Med 2002; 30: S281-S287.

$34 \mathrm{Lu}$ X, Garfield A, Rainger GE, et al. Mediation of endothelial cell damage by serine proteases, but not superoxide, released from antineutrophil cytoplasmic antibody-stimulated neutrophils. Arthritis Rheum 2006; 54: 1619-1628.

35 Speer $\mathrm{CP}$, Pabst MJ, Hedegaard $\mathrm{HB}$, et al. Enhanced release of oxygen metabolites by monocyte-derived macrophages exposed to proteolytic enzymes: activity of neutrophil elastase and cathepsin G. J Immunol 1984; 133: 2151-2156.

36 Aoshiba K, Yasuda K, Yasui S, et al. Serine proteases increase oxidative stress in lung cells. Am J Physiol 2000; 281: L556-L564.

37 Baird BR, Cheronis JC, Sandhaus RA, et al. $\mathrm{O}_{2}$ metabolites and neutrophil elastase synergistically cause edematous lung injury. J Appl Physiol 1986; 61: 2224-2229. 